DOI: 10.18027/2224-5057-2017-7-3s1-12-16

Цитирование: Петровский А. В., Амосова В. А., Хакуринова Н. Д. Циторедуктивные операции при метастатическом раке молочной железы // Злокачественные опухоли. - 2017. - Т. 7. - № 3, спецвыпуск 1. - С. 12-16.

\title{
Циторедуктивные операции при метастатическом раке молочной железы
}

\author{
А.В. Петровский', 2 В.А. Амосова', Н.Д. Хакуринова ${ }^{2}$ \\ 1 ФГБУ «НМИЦ онкологии им. Н. Н. Блохина» Минздрава России, 115478, Москва, Россия \\ ${ }_{2}^{2}$ ГБОУ ВПО «Первый МГМУ им. И. М. Сеченова» Минздрава России, 119048, Москва, Россия
}

Резюме: В данной статье представлен обзор современной научной литературы, освещающей особенности тактики лечения метастатического рака молочной железы. Еще несколько десятилетий назад данный диагноз представлял серьезную проблему и ассоциировался с меньшей продолжительностью жизни пациентов. В настоящее время достижения в лечении, в том числе в лекарственной терапии, позволили существенно повлиять на течение заболевания и улучшить прогноз и качество жизни. Однако вопрос о рациональной тактике ведения таких больных по-прежнему остается открытым.

Ключевые слова: метастатический рак молочной железы, комплексное лечение, хирургическое лечение

Рак молочной железы (РМЖ) является одной из ведущих проблем клинической онкологии у женщин (20,9\%): он занимает первое место по показателям заболеваемости (49,75 на 100 тыс. женщин - 66621 случай) и смертности (15,17 на 100 тыс. женщин - 23052 случаев). В настоящее время в России зарегистрированы 622978 больных РМЖ, их средний возраст - 61,3 года [1]. Примерно 8\% (около 7000 больных ежегодно) всех случаев впервые выявленного РМЖ - метастатические, причем буквально несколько десятилетий назад средняя продолжительность жизни таких пациенток не превышала 15 мес., более 3 лет удавалось прожить 15\% больных, а более 5 лет - только $10 \%$ пациентов [2-4], тогда как во всем мире этот процент равен $10,4 \%[5,6]$. Тем не менее, в настоящее время в России отмечается некоторое снижение смертности от РМЖ - стандартизированный показатель на 100 тыс. женского населения за период с 2005 по 2016 г. снизился на 12,53\% [1]. В значительной степени это объясняется достижениями последних лет в лечении РМЖ. Теперь средняя продолжительность жизни больных метастатическим РМЖ составляет 51 мес., а 3- и 5-летняя общая выживаемость при некоторых формах (метастазирование в кости, мягкие ткани) достигает $61 \%$ и $40 \%$ соответственно. Однако данные литературы, освещающие вопросы лечебной тактики при метастатическом РМЖ, свидетельствуют о большом разнообразии взглядов и отсутствии единого подхода к вопросу о необходимости удаления первичного очага. За последнее десятилетие стали появляться статьи, свидетельствующие об обоснованности хирургического лечения первичной опухоли у больных метастатическим РМЖ. В этих исследованиях проведена клиническая оценка значения удаления первичного очага у больных мета- статическим РМЖ и представлены доказательства целесообразности такого подхода.

Согласно многочисленным национальным и международным рекомендациям, основным методом лечения метастатического РМЖ является системная терапия (гормональная, таргетная, химиотерапия), которая может быть дополнена хирургическим удалением первичной опухоли с целью предотвращения различных осложнений, таких как изъязвление, кровотечение, боль. При этом оперативное вмешательство рекомендуется выполнять только при возможности полного удаления первичной опухоли и достаточной ожидаемой продолжительности жизни (т.е. при отсутствии отдаленных метастазов, представляющих угрозу жизни в ближайшее время). Альтернативным вариантом хирургического лечения в этой ситуации является лучевая терапия. Данные рекомендации основаны на результатах проспективных рандомизированных исследований, продемонстрировавших отсутствие положительного влияния хирургического лечения на общую выживаемость, в связи с чем удаление первичного очага не входит в принятые стандарты.

B 2015 г. R. Badwe и соавт. опубликовали результаты исследования NCT00193778, проведенного в Мумбае в 2005-2013 гг. На первом этапе всем пациенткам с метастатическим РМЖ проводили 6-8 циклов химиотерапии, из них $60 \%$ (415 женщин) ответили на данный вид лечения и были рандомизированы на 2 группы: в первой группе выполнялось хирургическое вмешательство, во второй нет. Медиана времени наблюдения составила 23 мес. Медиана продолжительности жизни составила 19,2 мес. в группе оперированных пациентов и 20,5 мес. - в контрольной группе (отношение рисков (ОР) 1,04; $95 \%$ дове- 
рительный интервал (ДИ) 0,81-1,34; p=0,79), т.е. хирургическое лечение не повлияло на общую выживаемость у данной группы больных. Кроме того, было отмечено, что даже при изолированном поражении костей и наличии менее 3 метастатических очагов оперативное вмешательство уменьшает продолжительность жизни, а также способствует дальнейшему развитию отдаленных метастазов (медиана продолжительности жизни 11,3 мес. у оперированных и 19,8 мес. у не оперированных (ОР 1,42; 95\% ДИ 1,08-1,85; $\mathrm{p}=0,012))$ [7].

Второе многоцентровое рандомизированное исследование (MF07-01) было проведено в Турции в 20072013 гг. Всем пациенткам, вошедшим в исследуемую группу, на первом этапе выполняли удаление первичной опухоли; 3-летняя выживаемость этих больных составила $60 \%$, тогда как у больных, получавших только системную терапию, $-51 \%(p=0,5)$. Отмечено также, что общая выживаемость (OB) оказалась на $34 \%$ выше в первой группе (OP 0,66; 95\% ДИ 0,49-0,88, p=0,005). Однако уже за 5-летний период наблюдения результаты существенно отличались и составили соответственно $41,6 \%$ у оперированных против 24,4\% у не оперированных пациенток (медианы общей продолжительности жизни - 46 и 37 мес. соответственно). Таким образом, с течением времени результаты в группе оперированных больных оказались значительно лучше, чем при проведении только системной терапии. При анализе результатов выявлено значительное увеличение общей выживаемости в группе хирургического лечения при положительных РЭ, РП (ОР 0,64, 0,46-0,91, p=0,01), отрицательном HER2 (95\% ДИ 0,38-0,86; $\mathrm{p}=0,006$ ), единичных метастазах в костях (ОР 0,47, 95\% ДИ 0,23-0,98; p=0,04), а также у больных моложе 55 лет. По мнению авторов, именно такие пациентки являются наилучшими кандидатами для удаления первичной опухоли [8].

В Университете Вирджинии А. M. Leung et al. [9] проанализировали истории болезней 157 пациенток, страдавших PMЖ IV стадии и получавших лечение в период с 1990 по 2000 г. Все больные были разделены на группы в зависимости от лечения: гормональная терапия, химиотерапия, лучевая терапия, хирургическое лечение и сочетание нескольких методов. Медиана продолжительности жизни пациенток, которым было выполнено удаление первичной опухоли, составила 25 мес., а у 105 (67\%) не оперированных больных - 13 мес. (log-rank p=0,06). Медиана времени жизни у 37 женщин, получивших только химиотерапию, составила 21 мес. по сравнению с 40 мес. у 14 больных, которым были проведены химиотерапия и лучевая терапия, и 22 мес. у 33 пациенток, получивших химиотерапию и хирургическое лечение. Однако эти различия оказались статистически не значимыми (log-rank p=0,36), скорее всего, из-за небольшого числа больных в сравниваемых группах. Многофакторный анализ показал, что единственным фактором, связанным с увеличением выживаемости, для всех групп больных является факт проведения химиотерапии ( $p=0,02)$, а не хирургического лечения.

В противоположность этому большое количество ретроспективных исследований продемонстрировали обратный результат: увеличение общей выживаемости больных метастатическим РМЖ при удалении первичного очага. Еще W. Halsted предположил, что РМЖ - локальная болезнь, и опухолевые клетки, отделяясь от первичного очага, дают начало отдаленным метастазам, что доказывает необходимость его удаления. В своей статье Ly B.H. и Nguyen N.P. [10], проанализировав 14 научных работ, касающихся этой проблемы, сообщили, что медиана общей продолжительности жизни увеличивается с 12,628,3 мес. среди пациенток, не получивших хирургического лечения, до 25-42 мес. у больных, у которых первичный очаг был удален.

В исследование, проведенное Gnerlich с соавт., были включены пациентки, данные о которых содержались в базе данных SEER (период с 1988 по 2003 г.). Проведение хирургического лечения у этих пациенток ассоциировалось с увеличением медианы продолжительности жизни. В результате пациентки, которым было выполнено удаление первичной опухоли, прожили на 11-17 мес. больше, чем не оперированные больные: медианы продолжительности жизни составили 36 и 21 мес. у оперированных и не оперированных больных соответственно $(\mathrm{p}<0,001)$. При этом вероятность смерти при удалении первичной опухоли снижалась в среднем на $37 \%$ [11].

Улучшение показателей выживаемости у оперированных больных демонстрируют и другие авторы. Blanchard c coавт. отметили, что время от постановки диагноза метастатического РМЖ до смерти пациента составило 27 мес. у оперированных больных и 17 мес. - у не оперированных [12].

В исследовании Ruiterkamp J. с соавт. при оценке 5-летней выживаемости 728 больных за период 1993-2004 гг. оказалось, что медиана продолжительности жизни оперированных пациенток $(\mathrm{n}=288)$ составила 31 мес., а не оперированных $(\mathrm{n}=440)$ - 14 мес. $(\mathrm{p}<0,0001) ; 5$-летняя общая выживаемость - 24,5\% и 13,1\% соответственно $(\mathrm{p}<0,0001 ; \mathrm{OP}=0,62 ; 95 \%$ ДИ=0,51-0,76). Кроме того, согласно опросу, оперированные пациентки чувствовали себя гораздо лучше и имели меньше сопутствующей патологии. При нормализации распределения пациенток по возрасту, количеству метастазов, видам лечения оказалось, что удаление первичной опухоли является независимым прогностическим фактором [13].

Khan S.A., Stewart A.K. и Morrow M. (National Cancer Database (NCDB) of the American College of Surgeons) проанализировали 3-летнюю выживаемость 16023 больных метастатическим РМЖ, получавших лечение в 1990 1993 гг., в зависимости от вида лечения и состояния краев резекции. Из них в 6861 (42,8\%) случае проводилась только системная терапия, в 9162 (57,2\%) случаях - операция и системная терапия. Трехлетняя общая выживаемость 
больных, получивших хирургическое лечение и системную терапию, при отсутствии опухолевых клеток в краях резекции $(\mathrm{n}=3396)$ составила $35 \%$, с наличием опухолевых клеток в краях резекции $(\mathrm{n}=2561)-26 \%$, в группе больных, получивших только системную терапию - 17,3\% ( $<<0,0001)$. Авторы сделали вывод о том, что общая выживаемость зависела не только от факта выполнения операции, но и от состояния краев резекции. Исследование также показало, что такие характеристики, как количество метастазов, их локализация и объем операции на первичной опухоли, являются важными независимыми факторами прогноза, а полное удаление первичного очага снижает риск летального исхода от РМЖ на $39 \%$ (OP=0,61; 95\% ДИ=0,58-0,65) [14, 15].

Аналогичные результаты получены Rapiti E. (Geneva Cancer Registry). Выделены основные прогностические факторы риска: биологические характеристики опухоли, количество отдаленных метастазов и «чистота» краев резекции. У больных метастатическим РМЖ при выполнении хирургического вмешательства с «негативными» краями резекции 5-летняя выживаемость без РМЖ достигла 27\%, при наличии опухолевых клеток в краях резекции - 16\%, в случаях с неизвестным состоянием краев резекции - $12 \%$ $(p=0,0002)$. При сравнении результатов лечения больных только с метастазами в костях и больных с поражением других органов оказалось, что удаление первичного очага снижает риск смерти во всех подгруппах, независимо от локализации метастазов. В стратифицированном анализе эффект был очевидным только у женщин с метастазами в костях: отношение рисков для этих больных оказалось равным 0,2 (95\% ДИ 0,1-0,4; $\mathrm{p}=0,001)$, у пациенток с метастазами в других областях - 0,7 (95\% ДИ 0,4-1,2). При этом выполнение подмышечной лимфаденэктомии не повлияло на течение заболевания [16].

В 2007 г. были проанализированы результаты лечения 409 пациенток за период с 1996 по 2005 г. При нормализации распределения больных по возрасту, соматическому статусу, размеру опухоли, гистологическому варианту и локализации метастатических очагов оказалось, что медиана продолжительности жизни пациенток, которым выполнили удаление первичной опухоли, была больше: 31,9 и 15,4 мес. соответственно ( $<<0,0001 ;$ ОР 0,53, 95\% ДИ $0,42-0,67)$. Было отмечено также статистически значимое влияние на выживаемость локализации метастазов: у больных с метастазами только в костях риск смерти оказался меньше (ОР 0,76; 95\% ДИ 0,58-0,98), а самый высокий риск был зарегистрирован в группе пациенток с висцеральными метастазами (ОР 4,57; 95\% ДИ 2,20-9,49) [17]. Это было подтверждено В. Cady с соавт. [18], которые проанализировали эффективность различных последовательностей системного и локального лечения у 808 женщин, страдающих РМЖ IV стадии. Наилучшие показатели общей выживаемости были достигнуты у пациенток с метастазами в костях, получивших химиотерапию на первом этапе, по сравнению с показателями больных, у которых системное лечение было начато после операции.

Вместе с тем в исследовании, проведенном в MD Anderson Cancer Center, статистически значимого увеличения общей выживаемости пациенток хирургической группы не обнаружено, при этом выживаемость без прогрессирования в этой группе больных была статистически значимо выше по сравнению с группой консервативной терапии [19].

Еще один важный вопрос, касающийся выполнения оперативного вмешательства у пациенток с метастатическим РМЖ - оптимальные сроки выполнения операции. Многофакторный анализ Rao R. с соавт. включал демографические данные, характеристики опухоли, локализацию метастазов, объем и сроки операции, лучевую и системную терапию. В зависимости от времени, прошедшего с момента диагностики метастатического РМЖ до хирургического вмешательства, все пациентки были разделены на 3 группы. Наилучшие результаты были зафиксированы при удалении первичной опухоли в сроки от 3 до 9 мес. от начала лечения [20].

Однако при многофакторном анализе было отмечено, что в хирургической группе преобладают пациентки более молодого возраста, что могло оказать влияние на прогноз и дальнейшие результаты. В исследовании Baylor College of Medicine больные, которым удалили первичную опухоль, были, наоборот, старше не оперированных пациенток. Из 16401 пациентки у 807 (4,9\%) был диагностирован рак молочной железы IV стадии, из них в 242 случаях $(61,3 \%)$ выполнено удаление первичной опухоли и 153 $(38,7 \%)$ больным хирургическое вмешательство не проводилось. Медиана продолжительности жизни в «хирургической» группе составила 27,1 мес. по сравнению с 16,8 мес. у больных, не получивших хирургического лечения ( $<<0,0001)$. В многофакторном анализе было доказано, что хирургическое вмешательство является независимым фактором, связанным с увеличением общей выживаемости $(p=0,006)$. Houston Blanchard D.K. с соавт. сообщают о том, что женщины, подвергшиеся хирургическому лечению, были значительно старше остальных, имели небольшие размеры первичной опухоли, меньшее количество метастазов, реже имели висцеральные метастазы. У этой категории пациенток также чаще регистрировались опухоли с положительными рецепторами [21].

В 2013 г. E. Harris и соавт. опубликовали метаанализ, включающий 10 публикаций, отобранных в MEDLINE. Обобщены результаты лечения 28693 больных метастатическим РМЖ, 52,8\% из которых подверглись удалению первичной опухоли. Отмечено статистически значимое увеличение 3-летней выживаемости в исследуемой группе (40 против 22\%; p<0,01). Наилучшие результаты после оперативного лечения наблюдались при меньшем размере первичной опухоли, менее значимых сопутствующих заболеваниях, одиночных очагах метастатического поражения. При этом зависимости эффекта лечения от локали- 
зации метастазов, биологических характеристик опухоли (степени гистологической злокачественности, экспрессии стероидных гормонов) выявлено не было [22].

Результаты наиболее крупных отечественных исследований по основным показателям совпадают с результатами приведенных зарубежных работ. В частности показано, что удаление первичной опухоли у больных первичным метастатическим PMЖ с ECOG 0-1 является независимым фактором прогноза и существенно увеличивает показатели выживаемости. Так, медиана продолжительности жизни оперированных пациенток (1-я группа) составила 51 мес., не оперированных (2-я группа) - 39 мес. $(p=0,054)$. Однолетняя общая выживаемость больных в 1-й группе достигла $83,1 \%$, во 2-й группе - 71,5\%, двухлетняя общая выживаемость - 71,1\% и 57,2\%, пятилетняя - 50\% и 33,8\% соответственно ( $p=0,054)$. Медиана времени до прогрессирования у больных, которым выполнялось оперативное вмешательство, достигла 27 мес., в группе без хирургического лечения - 11 мес. ( $\mathrm{p}=0,024) ; 1$-летняя выживаемость без прогрессирования составила 71,9\% и 40,2\%, 2-летняя - 56,2\% и 31,1\%, 5-летняя - 42,7\% и $21 \%$ в группах хирургического и консервативного лечения соответственно $(p=0,024)$. Таким образом, было показано, что удаление первичной опухоли улучшает отдаленные результаты лечения больных первичным метастатическим РМЖ. [23].

В 2016 г. были представлены результаты анализа отдаленных результатов лечения 608 пациенток с первичным метастатическим РМЖ по материалам 5 крупных онкологических центров. Установлено, что удаление первичной опухоли увеличивает медиану продолжительности жизни с 23 до 35 мес. (log-rank p<0,01); 3-летняя общая выживаемость увеличивается с $47 \%$ до $65 \%$, а 5 -летняя - с $21 \%$ до 45\% (log-rank p=0,001). Медиана времени до прогрессирования заболевания в исследуемой группе составила 12 мес., в контрольной - 8 мес. $(p<0,02)$. Наиболее эф- фективным удаление первичной опухоли было при локализации отдаленных метастазов в костях. При метастазах в костях 1-, 3- и 5-летняя выживаемость в группе больных, подвергшихся мастэктомии (n=113), составила соответственно 99\%, 71\% и 62\%, медиана продолжительности жизни - 45,5 мес. При 1-2 метастазах в единственном органе удаление первичной опухоли увеличивало медиану продолжительности жизни с 26 до 39 мес. ( $p=0,001)$, однако при $\geq 3$ очагах разницы в выживаемости между группами не отмечено $(p=0,07)$ [24].

\section{Выводы}

До настоящего времени вопрос о роли и времени выполнения операции, критериях подбора пациентов для удаления первичной опухоли при метастатическом РМЖ остается открытым. Многочисленные ретроспективные исследования указывают на пользу от проведения хирургического лечения и увеличение показателей выживаемости. Наиболее эффективно оперативное вмешательство при метастатическом поражении костей, 1-2 метастазах в пределах одного органа, наличии ответа на системное лечение, высокой экспрессии РЭ/РП, достижении «чистых» краев резекции. Существенно ухудшает прогноз и увеличивает риск смерти множественный характер поражения, висцеральные метастазы (особенно в печени), отсутствие ответа на системную терапию, отсутствие экспрессии РЭ/РП, опухолевые клетки в краях резекции. Только одно проспективное исследование демонстрирует противоположные результаты. В связи с этим необходимы новые проспективные рандомизированные исследования по оценке эффективности хирургических методик при лечении больных первичным метастатическим РМЖ.

\section{Литература • References}

1. Злокачественные новообразования в России в 2015 году (заболеваемость и смертность). Под ред. А. Д. Каприна, В. В. Старинского, Г. В. Петровой. М.: МНИОИ им. П. А. Герцена, 2017. 250 с.

2. Под ред. А.Д. Каприна, В.В. Старинского, Г. В. Петровой Состояние онкологической помощи населению России в 2015 году. М.: МНИОИ им. П. А. Герцена - филиал ФГБУ «НМИРЦ» Минздрава России, 2016. - илл. - 236 с. ISBN 978-5-85502-226-1.

3. Мерабишвили В. М. Выживаемость онкологических больных. СПб., 2011. С. 178, 261-4.

4. Семиглазов В. Ф., Нургазиев К.Ш., Арзуманов А. С. Опухоли молочной железы (лечение и просилактика). Алматы, 2001.

5. Fields R. C., Jeffe D. B., Trinkaus K. et al. Surgical resection of the primary tumor is associated with increased long-term survival in patients with stage IV breast cancer after controlling for site of metastasis, Time for more optimism in metastatic breast cancer?

6. Senkus E, Cardoso F, Pagani 0. Cancer Treat Rev. 2014 Mar;40 (2):220-8. doi: 10.1016/j. ctrv. 2013.09.015. Epub 2013 0ct 1. Review.

7. R Badwe, Rohini Hawaldar, Nita Nair, Rucha Kaushik, Vani Parmar, Shabina Siddique, Ashwini Budrukkar, Indraneel Mittra, Sudeep Gupta. Locoregional treatment versus no treatment of the primary tumor in metastatic breast cancer: an open-label randomized controlled trial Lancet Oncol 2015 Volume 16, No. 13, p1380-1388.

8. Randomized Trial Comparing Loco regional Resection of Primary Tumor with No Surgery in Stage IV Breast Cancer at the Presentation (Protocol MF07-01): A Study of Turkish Federation of the National Societies for Breast Diseases Atilla Soran, MD, MPH, 
FACS, * Serdar Ozbas, MD, Sheryl F. Kelsey, PhD, and Bahadir M. Gulluoglu, MD, FACS§ 2009 Wiley Periodicals, Inc., 1075-122X/09 The Breast Journal, Volume 15 Number 4, 2009399-403.

9. Leung A. M., Vu H. N., Nguyen K. A. et al. Effects of surgical excision on survival of patients with stage IV breast cancer. J Surg Res 2009;1-6.

10. Loco-regional treatment in metastatic breast cancer patients: Is there a survival benefit?/B. H. Ly, N.P. Nguyen, V. Vinh-Hung et al. // Breast Cancer Research and Treatment. - 2010. - Vol. 119. - P. 537-545.

11. Gnerlich J, Jeffe DB, Deshpande AD, Beers C, Zander C, Margenthaler JA. Surgical removal of the primary tumor increases overall survival in patients with metastatic breast cancer: analysis of the 1988-2003 SEER data. Ann Surg Oncol 2007;14:2187-94.

12. Blanchard DK, Bhatia P, Hilsenbeck SG, Elledge RM. Does surgical management of stage IV breast cancer affect outcome? (abstract 2110). Breast Cancer Res Treat 2006;100 (suppl 1):S118.

13. Ruiterkamp J. et al. Surgical resection of the primary tumor in stage IV breast cancer patients: Effect of favorable disease characteristics on surgical selection and outcome. 2009 Breast Cancer Symposium. Abstract № 227/L. Rivera, R. Chandrasekhar, G. Wilding et al. Does removal of the primary tumor in patients with metastatic breast cancer improve either local control or overall survival? San Antonio Breast Cancer Symposium: Abstract e11511/S. Samiee, P. Berardi, N. Bouganim et al. // Cancer Research. 2011. - Vol. 71, №24.

14. Khan, S. A. Does resection of an intact breast primary improve survival in metastatic breast cancer?/S. A. Khan // Oncology. - 2007. Vol. 21, № 8. - P. 924-932, 934, 942.

15. Khan, S. A. Does aggressive local therapy improve survival in metastatic breast cancer?/S. A. Khan, A. K. Stewart, M. Morrow // Surgery. - 2002. - Vol. 132 (4). - P. 620-627.

16. Rapiti E, Verkooijen HM, Vlastos G, et al. Complete excision of primary breast tumor improves survival of patients with metastatic breast cancer at diagnosis. J Clin Oncol 2006;24:2743-9.

17. Surgical resection of the primary tumor is associated with increased long-term survival in patients with stage IV breast cancer after controlling for site of metastasis/R. C. Fields, D. B. Jeffe, K. Trinkaus et al. // Annals of Surgical Oncology. - 2007. - Vol. 14 (12). - P. 3345-3351.

18. Cady B., Nathan N. R., Michaelson J. S. et al. Matched pair analyses of stage IV breast cancer with or without resection of primary breast site. Ann Surg Oncol 2008;15 (12):3384-95.

19. Babiera GV, Rao R, Feng L, et al. Effect of primary tumor extirpation in breast cancer patients who present with stage IV disease and an intact primary tumor. Ann Surg Oncol 2006;13:776- 782.

20. Timing of surgical intervention for the intact primary in stage IV breast cancer patients/R. Rao, L. Feng, H. M. Kuerer et al. // Ann. Surg. Oncol. - 2008. - Vol. 15 (6). - P. 1696-1702.

21. Association of surgery with improved survival in stage IV breast cancer patients/D. K. Blanchard, P. B. Shetty, S. G. Hilsenbeck, R. M. Elledge // Ann. Surg. - 2008. - Vol. 247 (5). - P. 732-740.

22. Harris E., Barry M., Kell M. R. Meta-analysis to determine if surgical resection of the primary tumor in the setting of stage IV breast cancer impacts on survival. Ann Surg Oncol 2013;20 (9):2828-34.

23. Чернова Е.В. и др. Целесообразность удаления первичной опухоли у больных с впервые выявленным диссеминированным раком молочной железы //Российский онкологический журнал. - 2013. - №. 2.

24. Оценка результатов НИР РООМ «Возможности хирургического лечения больных раком молочной железы IV стадии» А. А. Божок, Э. Э. Топузов, А. Д. Зикиряходжаев, А. С. Сухотько, В. А. Хайленко, Ю. С. Шатова, Н. А. Климов, Р. М. Палтуев. Опухоли женской репродуктивной системы 4»2016 Том12 с 17-24. 